\title{
The value of five - stage combined double - aortic arch incision in prenatal screening of low risk fetus
}

\author{
Shunan Zhang ${ }^{1}$, Chu Shen ${ }^{2 *}$ and Xin Zhang ${ }^{2}$ \\ ${ }^{1}$ Department of Pathology, Affiliated Hospital of Jiangsu University, Zhenjiang, China \\ ${ }^{2}$ Department of Ultrasound medicine, Affiliated Hospital of Jiangsu University, Zhenjiang, China
}

\begin{abstract}
Objective: To study how to check the difficulty of fetus heart examination in low risk fetus structure screening during the second trimester, and to explore how to use two-dimensional ultrasonography and color Doppler imaging to maximize the detection rate of fetal heart abnormalities. The more standardized, effective and rapid inspection methods.

Methods: Pregnant women who were screened for fetal structure during 18-24 weeks' gestation were enrolled in this study. Fetal heart was routinely screened at the third level using fetal two-dimensional section technique and color Doppler imaging. Fetal heart of each case using the "fetal heart ultrasound 5 section basic section combined with double-aortic arch section method" were observed. From the bottom up are: 1 , transverse section of the abdomen; 2 , dual inflow section; 3 , left ventricular outflow tract section; 4, right ventricular outflow tract section; 5, three vascular section; Discovery of Abnormal or Suspicious Anomalies Further append requests for this fetal line of echocardiography are directed to the fourth level of fetal heart screening. And some serious cardiac abnormalities or combined with multiple organ malformation fetus fetus specimens taken basic anatomical control analysis, and a large number of late follow-up control analysis.
\end{abstract}

Results: This method can effectively improve the detection rate of fetal heart abnormalities.

Conclusion: The method is used to instruct the ultrasound doctor to detect the fetal heart abnormality during the screening of the third-level structure of the fetus with low-risk during pregnancy, which can effectively reduce or avoid the missed diagnosis.

\section{Introduction}

In the mid-pregnancy screening of low-risk fetal structure, the detection of fetal cardiac structural deformity, whether or not missed as little as possible is a check in the focus and difficulty; and fetal heart deformity is the most common Of fetal malformations, and the incidence of fetal cardiac malformations was significantly higher than the average incidence of cardiac malformations in living infants $8 \%$, congenital heart disease is also the most common human congenital diseases [1,2]. Fetal heart disease is also more complicated and varied. A variety of cardiac malformations that can not survive after birth are likely to occur in the fetus, such fetuses may not completely structural deformation associated with other systems; the same time, due to fetal abdominal position is constantly changing, the examiner can not let the fetus fixed in a Individual position, resulting in fetal heart position of various parts of the space to grasp a certain degree of difficulty. Therefore, in the third level of screening of fetal structure, rather than for the fourth level of fetal heart check, check how doctors can quickly identify the existence of fetal heart problems, mid-fetal structure screening is the focus of fetal heart malformation detection. Especially when the fetal heart in the chest position, the size did not occur obvious abnormalities and four chambers can be seen, and other fetuses also found no abnormalities in other systems, there may be to check the doctor negligence on other key aspects of the requirements, which is the author emphasizes the need to observe To the original intention of five sections [3].

\section{Data and methods}

\section{General information}

Clinical cases: Pregnant women who volunteered for fetal structural screening between June 2010 and October 2017 were enrolled in the study. Fetal structure screening was performed in more than 24,200 gestational weeks at 18-25 weeks in our hospital. Since 2011, Summary experience began to use and promote the five sections of the doublearch method of fetal heart examination, and with the team using this method to check nearly 16,200 cases of fetuses, are found in any section of the suspicious abnormalities, that is, plus fetal heart The fourth level of screening, all cardiac malformation fetus after basic medical examination after induction of labor or postpartum follow-up.

Instrument and method: GE Voluson730, GE VolusonE10 ultrasonic diagnostic apparatus, Aiplo500 ultrasonic diagnostic apparatus, and the probe center frequency $4-6 \mathrm{MHz}$.

In the routine screening of tertiary structure of the fetus, the use of five sections of the fetal heart examination:

Correspondence to: Chu Shen, Department of Ultrasound medicine, Affiliated Hospital of Jiangsu University, No.438 Jiefang Road, Jingkou District, Zhenjiang, Jiangsu 2121001, PR China; E-mail: anemone430@163.com

Key words: fetal prenatal screening, five sections combined double-aortic arch section examination, fetal structure low-risk screening

Received: March 22, 2018; Accepted: April 16, 2018; Published: April 19, 2018 
Bottom up to check: 1, abdominal cross-section: This section mainly in the cross-sectional guidance of the spinal cord to quickly determine the fetal abdominal aorta, inferior vena cava, liver and venous catheter orientation is normal, the plane is relatively simple, Once the orientation is normal, it quickly moves to the next plane. 2, to the fetal head to move the probe looking for dual inflow tract: This plane is basically the same as the four chamber heart section, observe the fetal cardiothoracic ratio, apical point, the approximate ratio of left and right atrium, left and right ventricular approximate ratio, mitral, Whether the mitral valve is visible or not, the horizontal position of the heart, the presence or absence of echo in the upper and lower foramen ovale, the existence of interventricular septum, atrioventricular septa and atrioventricular valve cross, especially the use of color Doppler left and right The number of inflows, the direction of blood flow, and the presence or absence of regurgitant signals on the flap are all aspects of the fetal heart screening that must be examined. Abnormalities of this section include: left and right ventricular or left and right atrial asymmetry in size, two-dimensional and color Doppler cannot see the symmetrical dual inflow of blood flow, significant reflux signal appears on the flap, the cross is not complete, the pericardial cavity Liquid dark area width $>2 \mathrm{~mm}$. 3, left ventricular outflow tract section: on the basis of four-chamber heart-shaped probe to the apical side of the clockwise rotation obtained to observe the left ventricular outflow tract and ascending aorta connection and the width is normal, can clearly observed the movement of the aortic valve; In this section can also see the left atrium, mitral valve, ventricular septum, open color Doppler observation of left ventricular outflow tract blood flow direction and status; this section slightly deflected the fetal head side of the probe to see the three blood vessels flat. Abnormalities in this section include abnormalities in left ventricular outflow tract width, abnormal flow of color Doppler flow signals, and / or colorful and bright colors. Three vessels / three vascular anomalies / three vessels cannot be seen after deflection of the probe Arrangement of orientation, spacing disorder, can be initially judged as abnormal. 4, right ventricular outflow tract section: the left ventricular outflow tract section of the probe to the left ventricular long axis of the right ventricular side deflection available to observe the right ventricular outflow tract and pulmonary artery trunk connection and the width is normal, but also clearly observed Movement of the pulmonary valve, and even the left pulmonary artery can be seen at the bifurcation of the right pulmonary artery, open color Doppler observed right ventricular outflow tract to pulmonary artery blood flow and state; and here the probe in the left ventricular outflow tract and right ventricular outflow A slight deflection between the side of the road section to move, use this to determine the aorta and the main pulmonary artery between the initial angle is normal. Abnormal standard of this section: right ventricular outflow tract width anomalies, color flow direction, abnormal color, aorta and pulmonary artery between the normal loss of nearly 90 degrees of deflection angle, or the aorta and the main pulmonary artery have a larger width of the two contrast Differences are unusual. 5, three vascular section: left ventricular outflow tract slightly deflected to the fetal head side of the probe to see the three-vascular plane. Abnormalities include: the probe deflection cannot see the three blood vessels or see the four vessels or three vascular diameter ratio was abnormal (Figures 1-6), spacing disorders, can be initially determined to be abnormal. 6, double-arch long axis view: through the fetus abdomen or dorsal, along the fetus can be found along the direction of the sagittal plane aortic arch long axis, arterial catheter arch long axis images mainly observe the connection, continuous and the width of whether there is significant abnormalities, Le can help to observe the continuity of two bow. This section abnormalities, the majority of major vascular malformations, the need

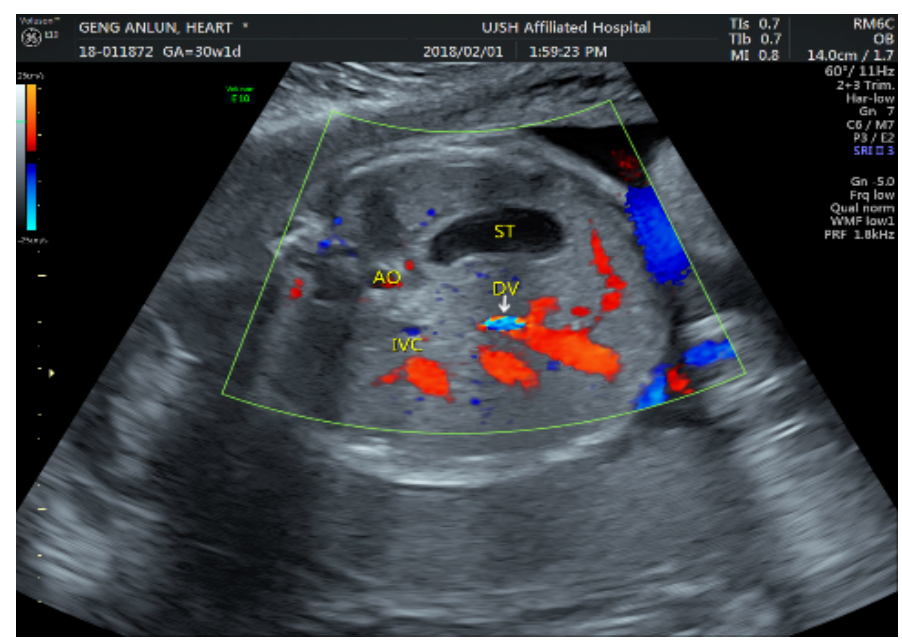

Figure 1. The first section of the abdomen

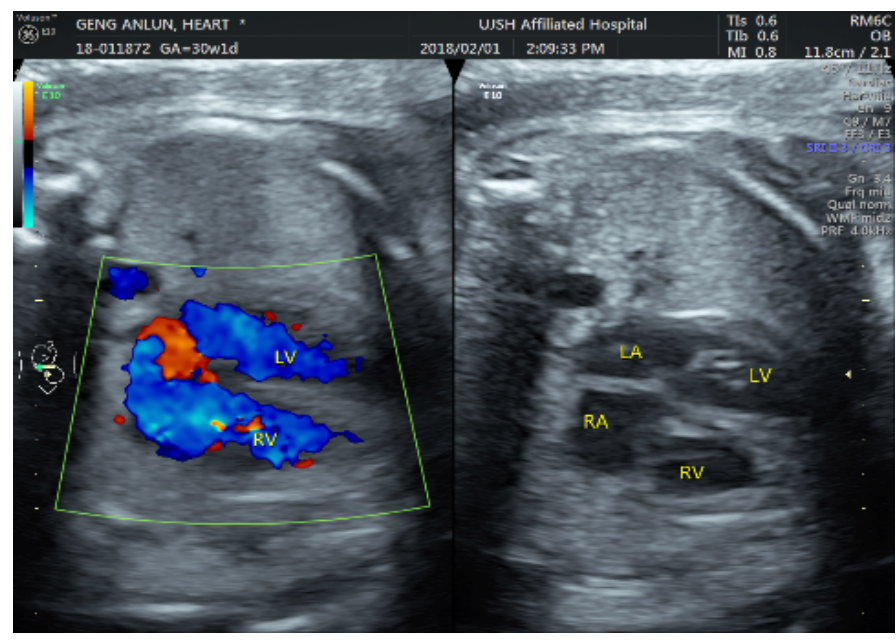

Figure 2. The second section of four chamber heart section

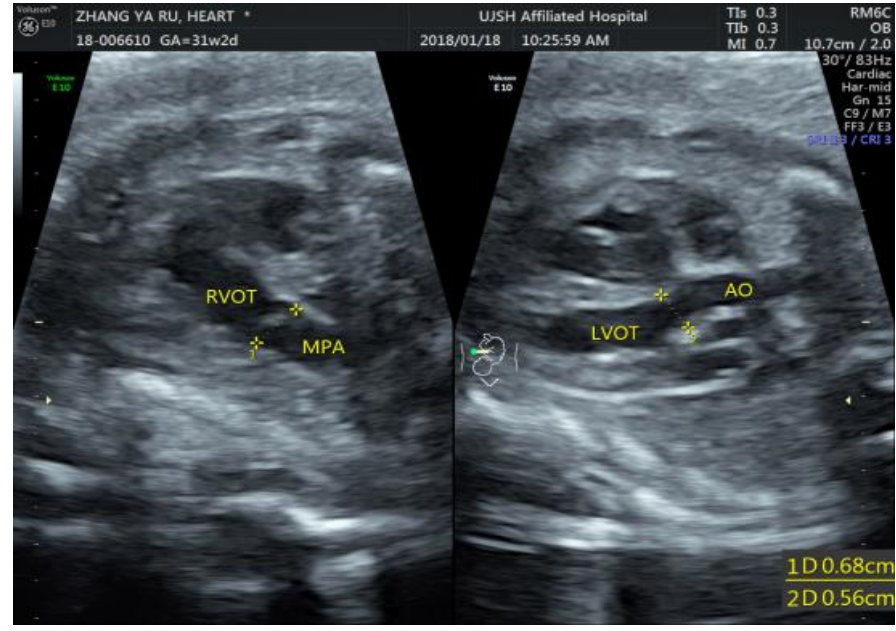

Figure 3. The third section left and right ventricular outflow tract section

for high priority, including: aortic arch, patent ductus arteriosus in any one of the two continuity showed difficulties or can not be displayed when the continuous, the color blood flow width inconsistencies, we must pay attention to the application Fetal heart of the fourth level of screening. 


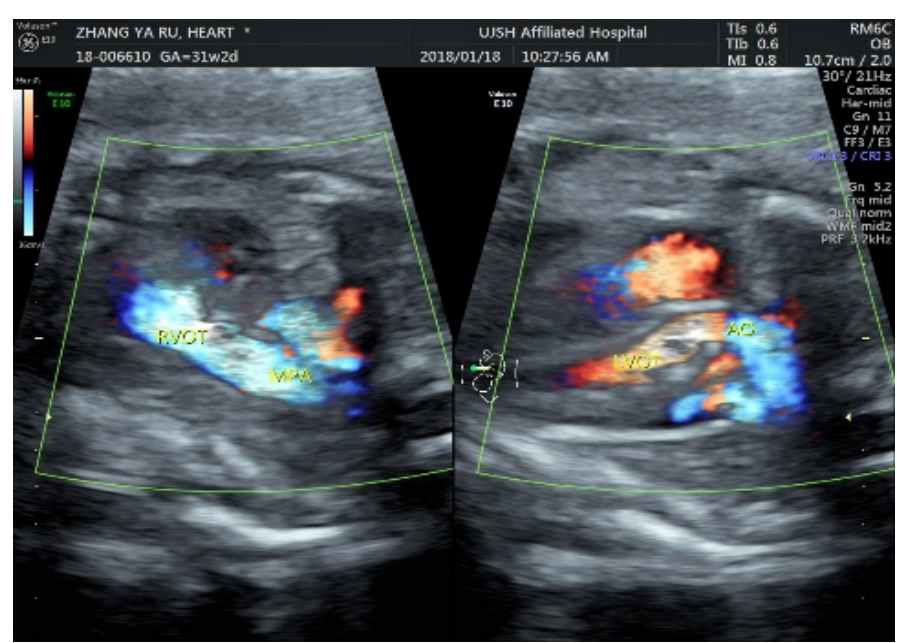

Figure 4. The fourth section of the three vascular sections

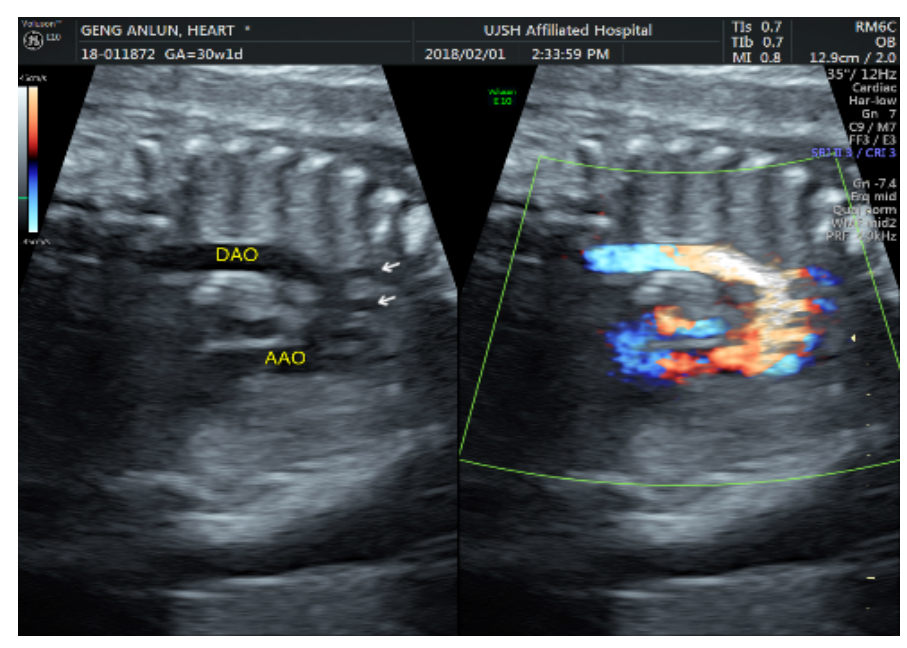

Figure 5. Aortic arch section

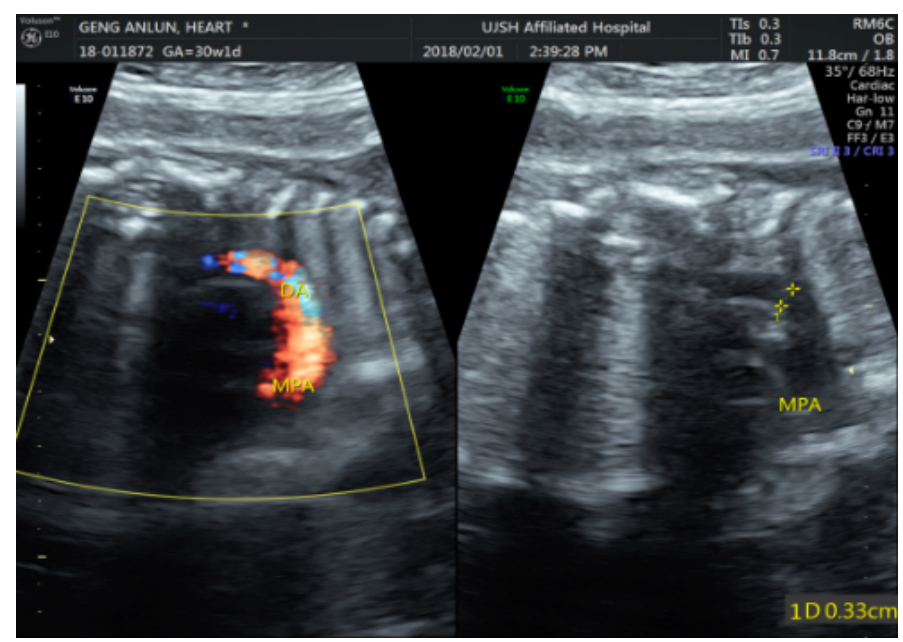

Figure 6. Artery catheter arch section

\section{Results}

The above screening time is generally 6-10 minutes. Approximately $2-6 \%$ of the more than 24,200 fetal exams are required for further fetal heart level 4 screening, most of which require examination due to bright spots of the heart and some require additional cardiac examinations due to non-cardiac heart failure, 28 cases of single umbilical artery or permanent right umbilical vein for routine heart examination, and the remaining 98 cases using five sections combined two bow cutting method analysis and found suspicious cardiac abnormalities, apply for the fourth level of fetal heart examination, 51 cases were confirmed cardiac malformations.

Among them, the first section of the abdomen found vascular abnormalities in the diagnosis of spleen-free syndrome in 1 case; the second section abnormalities were diagnosed in 3 cases of ventricular septal defect; atrial septal defect in 1 case; partial or complete and partial endocardial cushion defect 5 cases; left ventricular dysplasia in 5 cases *; single ventricle in 1 case; third section abnormalities, diagnosis of tetralogy of Fallot in 7 cases; right ventricular double outlet in 8 cases *; large vessel transposition in 4 cases *; 4 cases of arterial disease ${ }^{*}$; pulmonary valve stenosis in 1 case; the fourth paragraph found abnormalities, the simple diagnosis of left anterior superior left vena cava in 3 cases; double-arched arch found continuous abnormalities or difficulties in the diagnosis of aortic arch off 3 cases *; aortic arch narrowed in 3 cases; Pulmonary constriction, pulmonary valve atresia in 1 case; Another two cases, one case of 29 weeks of pregnancy was found to significantly increase the heart, tricuspid regurgitation, pericardial effusion, induced abortion after basic medical examination confirmed the absence of anatomical fetal heart anatomy, after further clinical diagnosis Virus infection caused by abnormal heart; the other case of pericardial effusion, two weeks after the pericardial effusion was significantly increased with significant abnormal cardiac function.

This method found suspicious abnormalities and four levels of cardiac screening, diagnosed as deformity detection rate increased year by year, from about $20 \%$ to nearly $40 \%$; and all cardiac malformation fetus after basic medical examination or postnatal follow-up Confirmed. In the screening of more than 16,200 cases of low-risk fetuses, which benefited from the "five-section combined double-aortic arch incision," no missed cases of important fetal malformations have been reported.

\section{Discussion}

Congenital heart disease is the most common fetal malformations, but also one of the major causes of neonatal deaths, once the misdiagnosis or missed diagnosis of a family and engaged in the screening work of the doctors themselves are more heavy blow; fetal structural screening responsibilities The existence of fetal heart structure problems has always put pressure on fetal structural screening, especially ultrasonographers who are unskilled in echocardiography. We hope that all doctors who carry out screening of fetal structures will be able to master this method provided by me. This method is rational and easy to remember, so that we can more surely screen the basic structure of the fetal heart Most cardiac suspicious abnormalities can be found, and the point is that abnormalities can be found. Once an abnormality is found, a further screening of the fourth level of the fetal heart for a fourth time based on the fetal condition is performed, and further systematic diagnosis of fetal cardiac malformations is performed by a physician qualified for echocardiography and / or fetal heart screening, Made a more definitive diagnosis. From the discovery of suspicious cardiac abnormalities to the diagnosis of the existence of the detection rate significantly increased the phenomenon, but also from another side reflects the five sections of the joint double-arch examination method with significant results. Of course, for the secondary secundum atrial septal defect, small ventricular septal defect, especially when the shunt is not obvious, hope in the fetal diagnosis, is still more difficult [3]. 
A review of several cases of misdiagnosis of cardiac malformations that occurred in the 2009-2017 screening effort occurred almost all before 2014. Approximately 10,000 low-risk fetal screening tests by 2014 did not warrant the use of this test. Including two cases of macrovascular malformations. Due to overreliance on the normal display of the four-chamber heart section, the double-aortic arch, ie, the aortic arch and the arterial catheter arch, has not been observed. However, when the aortic arch is severed, the four-chamber heart may completely disappear; A case of tetralogy of Fallot missed diagnosis because it failed to fully display the left ventricular outflow tract section, and lack of effective observation of left ventricular outflow tract connectivity, and color Doppler flow direction; also tells us in practice the fetus Screening, the four-chamber heart section is indeed important, but the screening of fetal heart has some limitations [4-7].

Color Doppler color flow examination is also an important means for the color Doppler to observe the presence or absence of a reflux signal on the atrioventricular valve of the dual inflow tract in the examination of each section. The left and right ventricular outflow tract blood flow Direction, nature is equally important. The aortic arch, ductus arteriosus blood flow signal continuity, is to find abnormalities such as aortic disconnection guarantee. We have seen a case of fetal pulmonary atresia of the fetus, is to find the opposite direction of the main pulmonary artery flow and diagnosis. Of course, the focus of this quick test is how to detect fetal heart abnormalities and ask questions to prevent misdiagnosis. Further definitive diagnosis, it is entirely possible to give the fourth level of fetal heart screening to do. The recommended range of gestational age for further four-stage screening of the fetal heart is 20 to 28 weeks. But even then, fetal echocardiography has limitations in some important related deformities and may sometimes require additional MRI to further confirm the extent of fetal heart malformation. Since the application of " 5 sections of the basic section combined with double-aortic arch incision method", in addition to atrial septal defect, patent foramen ovale has not yet been more serious missed diagnosis, especially in the past three years of practice more fully proved the use of This method can be very effective in avoiding and reducing missed diagnosis of fetal heart deformity.

\section{Notes}

1. The information does not include small atrial septal defect, patent foramen ovale, patent ductus arteriosus, and small ventricular septal defect with no obvious shunt signal.

2. this information fetal heart abnormalities in the four-screening detection rate analysis does not include cardiac tendons glare application examiner.

3. "*" marked cardiac abnormalities are mostly found in the heart of the four screening of the heart, blood vessels at several deformities.

\section{Acknowledgements}

This work was supported by a project of construction capacity for birth defect screening and diagnosis laboratory in Jiangsu (BM20150204), Social Development of Zhenjiang (SH2014032), the key project of health science and technology in Zhenjiang (SH2014084).

\section{References}

1. Li S (2006) Prenatal ultrasonographic diagnosis of fetal malformation[M]. Beijing, People's military medical publishing house 170 .

2. Hoffman JI, Kaplan S (2002) The incidence of congenital heart disease. J Am Coll Cardiol 39: 1890-1900. [Crossref]

3. Qiuyan Pei, Meiying, Jianguo Li, Xin Huang, Yanqiu Wei (2007). Importance of Fourchamber and Outflow Tract Views in Prenatal Screening for Congenital Heart Defects. Chinese Journal of Ultrasound in Medicine 7:538-540

4. Carvalho JS, Mavrides E, Shinebourne EA, Campbell S, Thilaganathan B (2002) Improving the effectiveness of routine prenatal screening for major congenital heart defects. Heart 88: 387-391. [Crossref]

5. Buskens E, Grobbee DE, Frohn-Mulder IME, Stewart PA, Juttmann RE, et al. (1996) Efficacy of routine fetal ultrasound screening for congenital heart disease in normal pregnancy. Circulation 94: 67-72. [Crossref]

6. Stumpflen I, Stumpflen A, Wimmer M, Bernaschek G (1996) Effect of detailed fetal echocardiography as part of routine prenatal ultrasonographic screening on detection of congenital heart disease. Lancet 348: 854-857. [Crossref]

7. Tegnander E, Eik-Nes SH, Linker DT (1994) Incorporating the four chamber view of the fetal heart into the second-trimester routine fetal examination. Ultrasound Obstet Gynecol 4: 24-28.

Copyright: (C)2018 Zhang S. This is an open-access article distributed under the terms of the Creative Commons Attribution License, which permits unrestricted use, distribution, and reproduction in any medium, provided the original author and source are credited. 